\title{
An Artificial Neural Network for the Selection of Winding Material in Power Transformers
}

\author{
Eleftherios I. Amoiralis, Pavlos S. Georgilakis, and Alkiviadis T. Gioulekas \\ Technical University of Crete, University Campus, Kounoupidiana, Chania, Greece \\ \{Eamoiralis, Pgeorg\} @dpem.tuc.gr
}

\begin{abstract}
The selection of the winding material in power transformers is an important task, since it has significant impact on the transformer manufacturing cost. This winding material selection has to be checked in every transformer design, which means that for each design, there is a need to optimize the transformer twice and afterwards to select the most economical design. In this paper, an Artificial Neural Network (ANN) is proposed for the selection of the winding material in power transformers, which significantly contributes in the reduction of the effort needed in the transformer design. The proposed ANN architecture provides $94.7 \%$ classification success rate on the test set. Consequently, this method is very suitable for industrial use because of its accuracy and implementation speed.
\end{abstract}

\section{Introduction}

In today's competitive market environment there is an urgent need for the transformer manufacturing industry to improve transformer efficiency and to reduce costs, since high quality, low cost products, and processes have become the key to survival in a global economy [1]. In addition, the variation in the cost of the materials has direct impact in the optimum transformer design. This work investigates the selection of the material of the transformer windings, which can be copper (CU) or aluminum (AL). Since CU and AL are stock exchange commodities, their prices can significantly change through time. Consequently, in some transformer designs, it is more economical to use CU windings instead of AL windings and vice versa. However, this has to be checked in every transformer design, optimizing the transformer twice and afterwards selecting the most economical design. In this paper, an ANN technique is applied to power transformers for the selection of the winding material. The proposed method is very fast and effective, which makes it very efficient for industrial use.

\section{Optimum Transformer}

This section describes the method for the determination of the optimum transformer, namely the transformer that satisfies the technical specifications [2] and the customer needs with the minimum manufacturing cost. In the industrial environment considered, three-phase wound core power transformers are studied, whose magnetic circuit is of shell type. The optimum transformer is calculated with the help of a suitable 
computer program, which uses 134 input parameters in order to make the transformer design as parametric as possible [3]. Among the acceptable solutions, the transformer with the minimum manufacturing cost is selected, which is the optimum transformer. It is important to note that some of these 134 input parameters have very strong impact on the determination of the optimum transformer such as the unit cost (in $€ / \mathrm{kg}$ ) of the magnetic material and the type of the winding material (CU or $\mathrm{AL}$ ).

\section{Proposed Methodology}

In this work, a fully connected three-layer feedforward ANN is trained in a supervised manner with the error back propagation algorithm [4]. This technique is applied to power transformers for the selection of the winding material, which is a classification problem [5] into two classes: CU or AL. In order to create the learning and test sets, 6 power ratings $(250,400,630,800,1000$ and $1600 \mathrm{kVA})$ are considered. For each transformer, 9 categories of losses are taken into account, namely $\mathrm{AA}$ ', $\mathrm{AB}$ ', $\mathrm{AC}$ ', BA', BB', BC', CA', CB', CC', according to CENELEC [2]. For example, a 250 KVA transformer with AC' category of losses has $3250 \mathrm{~W}$ of load losses and $425 \mathrm{~W}$ of no load losses [2]. Five different unit costs (in $€ / \mathrm{kg}$ ) are considered for the $\mathrm{CU}$ and the AL winding. Based on the above, 6.9.5 = 270 transformer design optimizations with $\mathrm{CU}$ winding and 270 transformer design optimizations with AL winding are realized. In total, $6 \cdot 9 \cdot 5^{2}=1350$ final optimum designs are collected and stored into databases. The databases are composed of sets of final optimum designs (FOD) and each FOD is composed of a collection of input/output pairs. The input pairs or attributes are the parameters affecting the selection of winding material. Thirteen attributes are selected based on extensive research and transformer designers' experience (Table 1). The output pairs comprise the type of winding (CU or AL) that corresponds to each FOD. The learning set is composed of 675 FODs and the test set has 675 FODs (different than the FODs of the learning set).

The number of neurons in the input layer is equal to the attributes, while the output layer comprises a single neuron, corresponding to the optimum winding material: $\mathrm{CU}$ or AL. We note that the input-output data are normalized by dividing the value of each attribute by its maximum value, contributing to the efficient ANN training [6].

Table 1. Thirteen attributes have been selected based on extensive research and experience

\begin{tabular}{llll}
\hline Symbol & Attribute Name & Symbol & Attribute Name \\
\hline$I_{1}$ & CU unit cost $(€ / \mathrm{kg})$ & $I_{8}$ & Guaranteed winding losses $(W)$ \\
$I_{2}$ & AL unit cost $(€ / \mathrm{kg})$ & $I_{9}$ & $I_{7} / I_{8}$ \\
$I_{3}$ & $I_{1} / I_{2}$ & $I_{10}$ & Rated power $(\mathrm{kVA})$ \\
$I_{4}$ & $F E$ unit cost $(€ / \mathrm{kg})$ & $I_{11}$ & Guaranteed short-circuit voltage $(\%)$ \\
$I_{5}$ & $I_{4} / I_{1}$ & $I_{12}$ & $I_{7} / I_{10}$ \\
$I_{6}$ & $I_{4} / I_{2}$ & $I_{13}$ & $I_{8} / I_{10}$ \\
$I_{7}$ & Guaranteed $F E$ losses $(W)$ & \\
\hline
\end{tabular}




\section{Results and Discussion}

A wide range of different $\mathrm{ANN}$ architectures and training parameters were considered using the MATLAB ANN toolbox [7]. The number of hidden layers and the numbers of neurons in each hidden layer are parameters to be defined by trial and error. Nevertheless, it has been observed that in most applications, one hidden layer is sufficient [8]. In our method, one hidden layer is chosen. The number of the neurons in the hidden layer was varied from 7 to 39 (Fig. 1). After numerous experiments, the ANN with 13 hidden neurons was found to be sufficient for this work with favorable good results. In Fig. 1 we show the average accuracy (comes from ten different executions) of the learning and test set for each experiment. We note that the 13-13-1 topology (13 input neurons, 13 neurons in the hidden layer, and 1 neuron in the output layer) reaches the highest classification success rate on the test set, despite the fact that the learning set is not as good as the others.

In addition, a wide range of different transfer functions and training functions are used, taken from MATLAB ANN toolbox [7]. Thus, the log-sigmoid transfer function is used, which may have any value between plus and minus infinity, and squashes the output into the range 0 to 1 (modeled as logsig in MATLAB). In addition, a network training function is used that updates weight and bias values according to the conjugate gradient backpropagation with Polak-Ribiere updates (modeled as traincgp in MATLAB [6]) [9]. This function is capable of training any network as long as its weight, net input, and transfer functions have derivative functions. We note that all the experiments use maximum numbers of epochs equal to 100.

Based on the above, the optimal architecture for the proposed feedforward ANN [10] was reached after enough experimentation with various combinations structured as 13-13-1. In this case, the classification success rate on the learning set is $97.5 \%$ and $94.7 \%$ on the test set. It should be noted that the percentage of the learning and test set resulted in the average of ten different executions of the algorithm. This result is improved by $2.1 \%$ in comparison with the classification success rate obtained using decision trees [11].

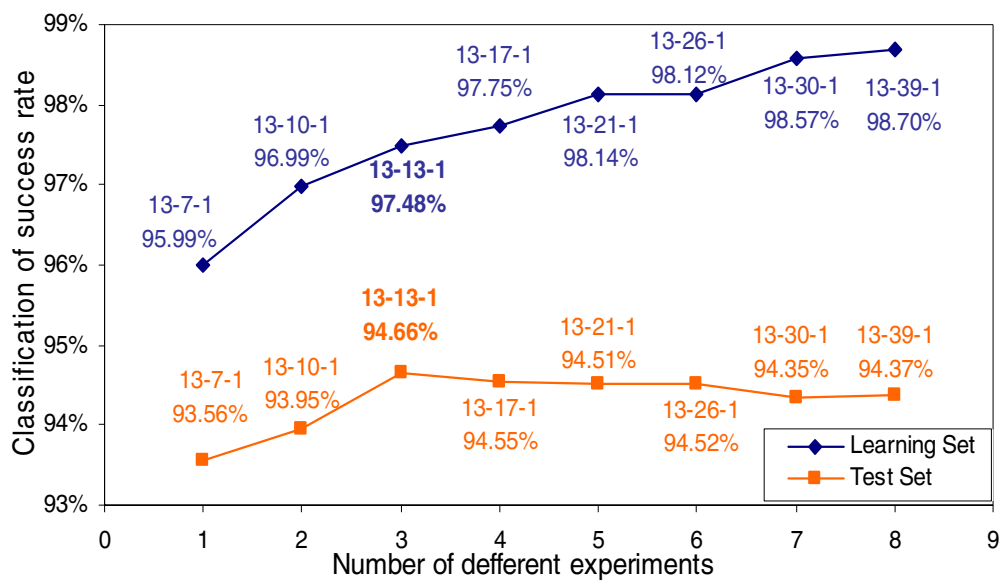

Fig. 1. Accuracy of each of the eight different topologies concerning the learning and test set 


\section{Conclusions}

In this work, a fully connected three-layer feedforward ANN was proposed in order to select the winding material in power transformers. The knowledge base was composed of 1350 final optimum designs. Half of them composed the learning set and the rest the test set. Thirteen attributes were selected based on extensive research and transformer designers' experience, and afterwards, were used as inputs in the ANN. The output pairs comprised the type of winding (CU or AL) that corresponds to each FOD. The performance of the ANN was found to be exceptional, which emerged this method as an important tool for classification. Specifically, the classification success rate on the learning set was $97.5 \%$ and $94.7 \%$ on the test set. The proposed method is very suitable for industrial use, because of its accuracy and implementation speed, since the ANN method eliminates the need to optimize the transformer twice.

\section{References}

1. Georgilakis, P., Hatziargyriou, N., Paparigas, D.: AI Helps Reduce Transformer Iron Losses. IEEE Computer Applications in Power 12 (2005) 41-46

2. CENELEC harmonization document 428.1 S1, (1992)

3. Georgilakis, P.S., Tsili, M.A., Souflaris, A.T.: A heuristic solution to the transformer manufacturing cost optimization problem. Proceedings of the 4th Japanese-Mediterranean Workshop on Applied Electromagnetic Engineering for Magnetic, Superconducting and Nano Materials (JAPMED'4), Cairo, Egypt, (2005)

4. Haykin, S.: Neural Networks: A Comprehensive Foundation, 2nd edn, Prentice Hall, (1999)

5. Zhang, G.: Neural Networks for Classification: A Survey, IEEE Trans. on Systems, Man, and Cybernetics 30 (2000) 451-462

6. Moravej, Z., Vishwakarma, D.N., Singh, S.P.: ANN-Based Protection Scheme for Power Transformer. Elect. Mach. Power Syst. (2000) 875-884

7. Denuth, H., Beale, M.: Neural Network Toolbox. For Use with MATLAB, User's Guide, Version 4.0, March (2005)

8. Coury, D.V., Jorge, D.C.: Artificial Neural Network Approach to Distance Protection of Transmissions Lines. IEEE Trans. on Power Delivery, 13 (1998) 102-108

9. Scales, L. E.: Introduction to Non-Linear Optimization. Springer-Verlag, New York (1985)

10. Fine, T.: Feedforward Neural Network Methodology. 1st edn, Springer-Verlag, (1999)

11. Georgilakis, P., Gioulekas, A., Souflaris, A.: A Decision Tree Method for the Selection of Winding Material in Power Transformers. Proceedings of the 4th Japanese-Mediterranean Workshop on Applied Electromagnetic Engineering for Magnetic, Superconducting and Nano Materials (JAPMED’4), Cairo, Egypt, (2005) 